\title{
Pharmaceutical Care in Homeopathic Treatment
}

\author{
Luciana Ferreira Mattos Colli and Palloma Valle Napolitano \\ Faculdade de Ciências da Saúde, Universidade do Grande Rio, Duque de Caxias - RJ 25071-202, Brasil
}

\begin{abstract}
Homeopathy is a therapy based on the principle of similarity. This therapy was proposed by the father of medicine, Hippocrates for almost 3000 years, thus developing the principle "Similia similibus curantur". Therapy with homeopathic medicines is widely used in therapeutics, but the patient often has difficulty in understanding how the treatment should be conducted. The Pharmaceutical Care, one pact relationship between patient and pharmacist, presents itself as an appropriate tool to assist in obtaining positive results in homeopathic therapy. Objectives: to demonstrate how the pharmacotherapeutic follow-AFT assists in detecting and solving problems related to homeopathic medicine and promotes improvements in response to treatment. Methodology: we performed literature review of the portals: SCIELO, MEDLINE and Google academics. Patients were interviewed by pharmacotherapeutic monitoring that was conducted-AFT employing the Dader method adapted to pharmaceutical care in homeopathic medicines in a pharmacy, this place was located in Rio de Janeiro, from November-December 2010 and January 2011. The research was previously approved by the Research Ethics Committee of the Unigranrio (CAAE: 4531.0.000.317-10). Results: the first of three interviews were identified DRM (drug related problem), having been required to apply IF-pharmaceutical intervention to adjust the behavior of the patient regarding the treatment and improvement of the results. Conclusions: the pharmacotherapeutic follow-AFT-introduced himself as appropriate methodology for managing patients on therapy with homeopathic medicines, and an improvement factor in the outcome of treatment of the patients.
\end{abstract}

Key words: Homeopathy, pharmaceutical care, interview, treatment.

\section{Introduction}

Homeopathy is a therapy based on the principle of similarity. This therapy was proposed by the father of medicine, Hippocrates for almost 3000 years, which was based on his observations, showed that it was appropriate to discuss, the contrary or the similar, whatever harm and from where it came. Thus develop the principle "Similia similibus curantur" is among others.

However, this therapy was founded by Samuel Hahnemann (1755-1843) who, when reading essays and articles began to test homeopathy on its own, and homeopathy evolved and developed until we reached the first Homeopathic Pharmacopoeia in 1805 [1] . So this therapy relies on the observation that any substance capable of causing certain symptoms in a healthy person can cure those same symptoms in a sick person [1].

Corresponding author: Luciana Ferreira Mattos Colli, M.S., research fields: pharmaceutical care.
Because homeopathy is a therapy based on the principle of similarity, where healing occurs by a similar agent to the pathology and which arises from the secondary reaction of the organism to the drug substance that observed patient's difficulty in understanding how the treatment will lead to results expected previously [2].

The Homeopathic treatment is prescribed by a homeopathic doctor and the drug is prepared by a pharmacist specialist who dispense the product should carry the pharmaceutical orientation, and develop the pharmacy Pharmaceutical Care.

The term Pharmaceutical Care was initially described as "a pact relationship between a pharmacist and a patient, in which the pharmacist carries out the functions of control and use of drugs, governed by conscience and commitment of interest in the patient.” This description led to conceptualization of pharmaceutical care as "the responsible provision of pharmacotherapy in order to obtain definite results that improve the quality of life of patients” [3]. 
A method of conducting the Pharmaceutical Care is Pharmacotherapeutic Tracking Dader method that was designed by the "Group of Research in Pharmaceutical Atención de la Universidad de Granada” in 1999.

The DRPs (Drugs Related Problems) are health problems, understood as negative clinical, pharmacological treatment that derivatives produced by various causes has as a consequence, not achieving the desired therapeutic goal or the appearance of side effects [3]. There are six types of DRPs: 1 - the patient has a health problem by not using pharmacotherapy you need, 2-the patient has a health problem by using a drug that does not need; effectiveness: 3 -the patient has a health problem for a non-quantitative ineffectiveness of pharmacotherapy, 4-the patient has a health problem for a quantitative ineffectiveness of pharmacotherapy and safety: 5-the patient presents a health problem by a non-quantitative uncertainty of a drug, 6- the patient presents a health problem for a quantitative uncertainty of a drug (Table 1) [4].

The Dader method of AFT - pharmacotherapeutic follow, Pharmaco Monitoring is a methodology created by a group in Granada University (Spain) and consists of a set of procedures to properly conduct the pharmaceutical care, where each professional, patient and doctor decide what to do in each particular case. The AFT - pharmacotherapeutic follow procedure has well-defined phases, which are structured in: provision of the service, the first interview, condition of situation, study phase, the evaluation phase, intervention phase, a result of the intervention, new state of the situation and successive interviews $[4,5]$.

The service begins with planning, the pharmacy must have a specific and private place for the execution of pharmaceutical care, then use the Dader method the patient will receive the invitation to participate, this invitation may occur in the pharmacy's store, often in a moment that the patient has doubts about his treatment or still some complaint about it. At the time of the invitation is scheduled date and time for the first interview [4].
At the first interview the environment should be adequate, quiet and there are no interruptions, the pharmacist should show interest in the patient's report. The pharmacist at this stage will be concerned with the patient's health problems, then what medication is used. At this point it is important to consider that many patients find it difficult to memorize the names of the medicines they use, many even report as follows: "This is the pressure ...", "the other is that of the stomach ..." Reporting in this way often becomes ineffective. A better option is to ask the patient at the time of the appointment, that she bring all the medicines she uses, or even their packaging [4-8].

The later steps of the Dader Method consist of defining the health status of the patient, as the patient is responding to the treatment. In the phase of study and evaluation the treatment is evaluated, characterizing the phase of AFT - pharmacotherapeutic follow up, and correlated to the state of health of the patient, and at this moment we can identify or not the occurrence of DRP, being necessary to IF-Pharmaceutical Intervention [4-8].

Sometimes during the pharmaceutical care procedures it will be necessary to take the pharmaceutical intervention, which is a documented act performed with the user and health professionals, aimed at solving or preventing DRPs [9]. The pharmacist should be aware of the need to have pipeline protocols in cases where the patient may present an adverse reaction to the treatment and prompt action will prevent injury or even represent therapeutic gain.

Considering that homeopathic treatment on the patient, questioning as an indication, dose, dosage, onset of therapeutic effect, and that this factor often leads it to leave the treatment [10]. Idealizing, enabling and implementing the AFT-pharmacotherapeutic monitoring for these patients will contribute to improving the quality of life and the same treatment through the identification, resolution, prevention of problems related to the use of the drug safety and 
effectiveness.

\section{Aims}

This study is aimed to demonstrate how the pharmacotherapeutic follow-AFT assists in detecting and solving problems related to homeopathic medicine and promoting improvements in response to treatment.

\section{Materials and Methodology}

\subsection{Bibliographic Review}

A bibliographic survey was carried out including books, articles and the literature on the portals: SCIELO, MEDLINE and Google academics. The keywords used were: homeopathy, pharmaceutical care, interview, treatment. Articles that were within the scope of the paper were considered.

\subsection{Pharmaceutical Care Service}

A qualitative field research was carried out, whose objective was to observe the main peculiarities of a pharmaceutical care service that was structured inside a pharmacy. The research group consisted of 10 (ten) patients in the age group of 25-60 years in treatment with homeopathic medicine.

The pharmaceutical care approach was carried out by the authors of the present study, the team was composed of a pharmacy academic and the guiding teacher, who is a pharmaceutical. The place went to a homeopathic pharmacy located in Rio de Janeiro, duly legalized next to the sanitary surveillance, in the period of Nov.-Dec. 2010 and Jan. 2011. The research was previously approved by the Ethics and Research Committee of the University of Grande Rio-Prof. José de Souza Herdy (CAAE: 4531.0.000.317-10 CEP-Research Ethics Committee/UNIGRANRIO).

The study was carried out using the Dáder method previously adapted according to the needs and profile of the establishment where the study was carried out. The following steps were performed: service offer, first interview, situation status, study phase, evaluation and intervention. Formula Certa ${ }^{\circledR}$ Alternate Technologies software was used to document and manage attendance.

The patients were invited to participate in the study, the invitation occurred in the store, at the moment the patient came to request the request for preparation of the drug or withdraw it. Of the 20 (twenty) invited patients only 10 (ten) accepted to participate in the study, and maintained frequency until the end of the evaluation.

Patients were scheduled to attend the pharmacy for the first interview, at which time the functionality of the Formula Certa ${ }^{\circledR}$ software was used, and the patient profile was recorded, as relevant information was recorded: weight, height, blood pressure at the moment of care, glucose, and additional information was requested such as: smoking, presence of chronic diseases. All medicines used by patients, including homeopathic and allopathic medicines, were surveyed. As a sequel to the interview the treatment was assessed and identified the main DRMs.

\subsection{Primer Explaining the Homeopathic Treatment}

An explanatory booklet was elaborated, containing some peculiarities of the homeopathic treatment, forms and schedules for the use of the drug, for the individuals participating in the study and other users of the pharmacy (Annex I). The explanatory booklet was elaborated from the evaluation of the patients and identification of the main doubts about the homeopathic treatment

\section{Results and Discussion}

The calls were made to customers in attendance and dispensing of pharmacy, characterizing the service offering phase. Patients in the study were those who showed more openness to join the program or to submit questions about the treatment, and they accepted readily participate. The number total of patients was ten (10).

The interview was scheduled to return the patient to establish, the reason for the choice is that it has been 
demanded by the patients themselves be the best time, requiring no additional cost to displacement.

The first interview was conducted as Dader method guides without form, questions were free and registered in accordance with the information given. At the time of the first interview, the patient data recorded on the property were checked by Formula Certa ${ }^{\circledR}$ Alternate Technologies program, and were considered mainly: compounded drugs used homeopathic and allopathic; and prescribing treatment time. Additional questions were asked to the patient, especially if he/she wore any industrialized medicine, thus complementing the information obtained through the software.

It was found during the provision of pharmaceutical services that all participants had problems with the amount of homeophatic medicine used and the frequency with which they were administered (Table 1).

After the first interview data were compiled and analyzed to establish the state of phase situation, study and evaluation. To frame the data were performed crossing the data collected with the classification of the Third Consensus of Granada (Table 2).

The DRPs are six, and are divided into problems with pharmacotherapy related to necessity, effectiveness and safety, during the pharmacotherapeutic follow-up of the patients, three (3) DRMs were identified, being the first problem identified was caused by necessity: the patient did not use a medication that is needed, this in the present study was triggered by the patient's forgetfulness regarding the times of use of the drug, forgetting being pointed as the main factor to cause this DRM (Table 2). Another DRM identified was in relation to the effectiveness, the patient demonstrated in the interview that the therapy was ineffective in a non-quantitative way, a significant number of patients decided to discontinue the treatment, without previous evaluation of the physician, assuming an improvement of the symptoms. And lastly, safety-related DRM was identified, and the observed was that the patient discontinued the treatment because he judged that the results of the treatment are delaying.

In the state of phase situation, study and evaluation concluded that the main DRPs observed occurred for lack of information and patient's awareness of the homeopathic therapy and its particularities, thus the IF-Pharmaceutical Intervention was focused on awareness, applying a brochure (Annex I) and AFT_pharmacotherapeutic follow (Fig. 1).

Table 1 Problems with patients at the first approach of pharmaceutical care.

\begin{tabular}{ll}
\hline Problems & \\
\hline Number of different drugs used; & $\begin{array}{l}\text { Prescription reading difficulty, if necessary, at home; } \\
\text { Patient with concept formed the treatment takes long time to } \\
\text { present results; }\end{array}$ \\
$\begin{array}{ll}\text { Omission to take, loss of time, times a day the medicine should be } \\
\text { taken; }\end{array}$ & $\begin{array}{l}\text { Motivation to self-medicate with claim that homeopathic } \\
\text { medicine offers no risk; } \\
\text { Interruption of treatment after feel improvement of symptoms; }\end{array}$ \\
\hline
\end{tabular}

Table 2 DRPs found after the first interview adapted from the Second Consensus of Granada in 2007.

\begin{tabular}{l}
\hline Necessity \\
DRP 1: the patient has a health problem by not using pharmacotherapy you need. \\
Major factor: by forgetting to take your medicine. \\
\hline Effectiveness \\
\hline DRP 3: the patient has a health problem for a non-quantitative ineffectiveness of pharmacotherapy. \\
Major factor: discontinue treatment to feel symptom improvement. \\
\hline Safety \\
DRP 5: the patient presents a health problem by a non quantitative uncertainty of a drug. \\
Major factor: delay of treatment in presenting effect.
\end{tabular}




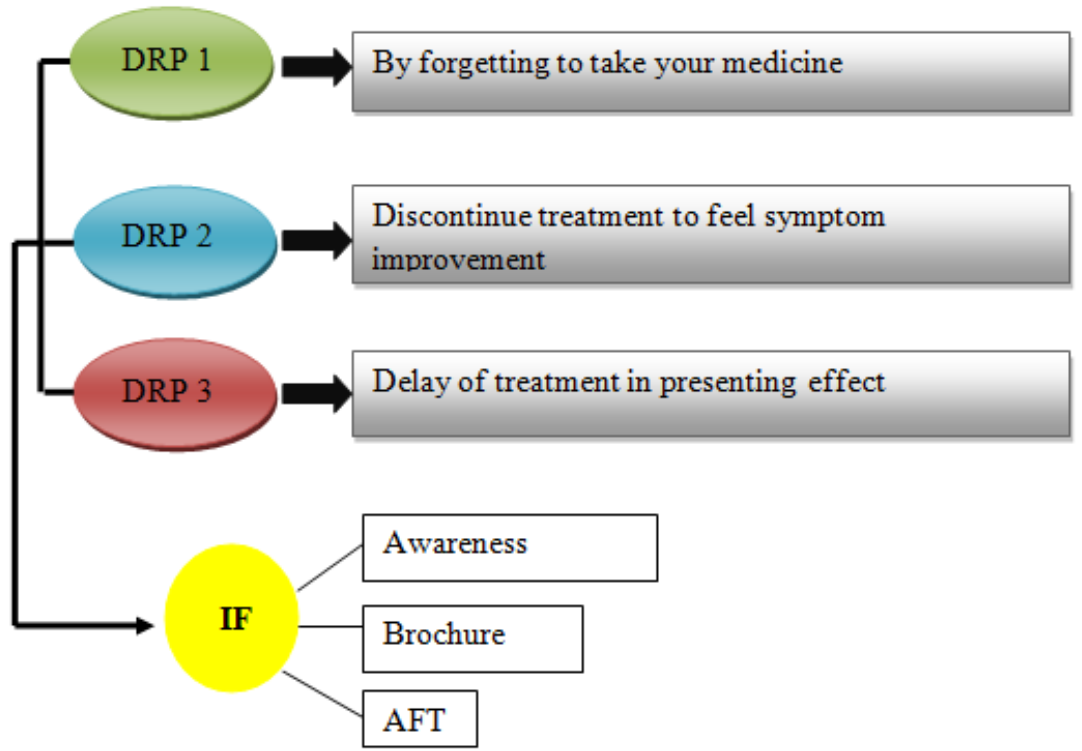

Fig. 1 Status situation, study and evaluation conducted with patients. DRP; IF-pharmaceutical intervention and AFT-pharmacotherapeutic follow.

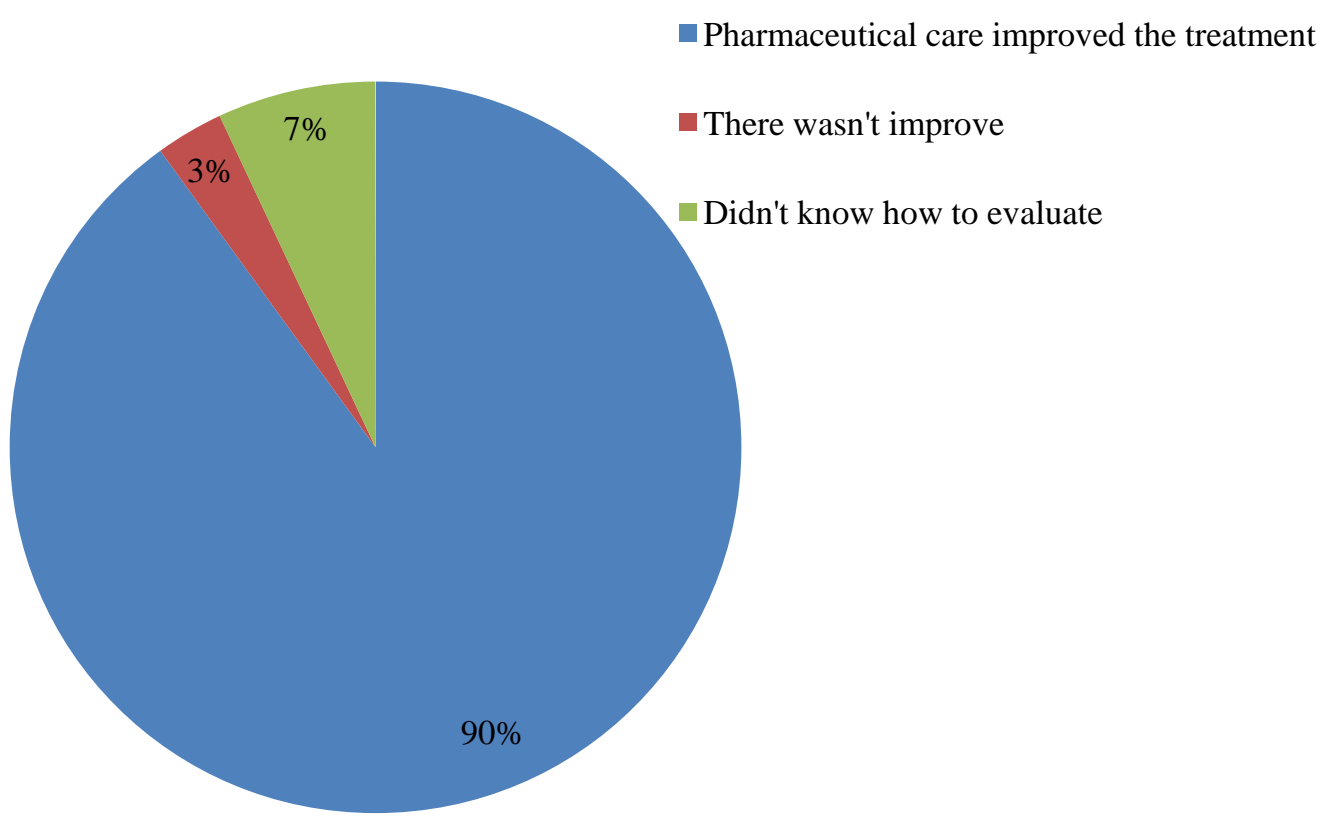

Fig. 2 Benefits of pharmaceutical care in the homeopathic treatment.

The second contact was made delivery of the brochure (Annex I), and awareness of patients regarding the particularities of homeopathic treatment.

By doing the third contact with the participants, $90 \%$ of patients reported that the service was of great help to determine schedules, information and answer questions about the medicine, and homeopathic treatment (Fig. 2). Thus, after the pharmaceutical intervention with the strategies of clarification to the patient, pharmacotherapeutic follow-up and distribution of primer the treatment evolved satisfactorily. 


\section{Conclusions}

This study showed that Dader method presented as suitable tool to implement pharmaceutical care focused on patients using homeopathic medicines, and that the pharmacotherapeutic follow-AFT was important to increase adherence to homeopathic treatment and promote improvement in the expected result.

Further studies in the future will have a great contribution to the topic, since the number of establishments that dispense homeopathic medicines is significant, and that the supply of pharmaceutical attention is increasingly present, being a reality in the professional activity of the pharmacist, who must be prepared, trained and able to perform the service.

\section{References}

[1] Hahnemann, S. 2006. "On the Power of Small Doses of Drugs in General and in Particular Belladonna.” In Minor Writings of Samuel Hahnemann, edited by R. E. Dudgeon. São Paulo: Organon.

[2] Morais, J. 2002. "The Power of Balls." Magazine Superinteressante n. 172, São Paulo: Publishing house Abril.

[3] Hepler, C. D., and Strand, L. M. 2007. Oportunidades y responsabilidades en la Atención Farmacéutica. Pharmaceutical Care España.

[4] Machuca, M., Fernádez-Llimós, F., and Faus, M. J. 2003. Dader Method: Pharmacotherapeutic Follow Instructions. Granada: GIAF-UGR.

[5] Yokoyama, C. S., et al. 2011. "Proposta de sistema de informação para atenção farmacêutica baseado no Método Dáder." Revista de Ciências Farmacêuticas Básica e Aplicada 32 (1): 19-26.

[6] American Society of Healthy-System Pharmacists. 2003. "ASHP Guidelines on Documenting Pharmaceutical Care in Patient Medical Records." American Journal of Health-System Pharmacy 60: 705-7.

[7] Gloria Nichols-English, P. S. 2000. "Optimizing Adherence to Pharmaceutical Care Plans.” Japha-Washington 40 (4): 475.

[8] Farris, Karen B., Fernandes-Llimos, F., and Benrimoj, S. I. 2005. Pharmaceutical Care in Community Pharmacies: Practice and Research from around the World.

[9] Santos, P. C. J. 2016. Atenção Farmacêutica: contexto atual, exames laboratoriais e acompanhamento farmacoterapêutico. São Paulo: Editora Atheneu, p. 5.

[10] Fontanella, F., Speck, F. P., Piovezan, A. P., and Kulkamp, I. C. 2007. "Knowledge, Access and Acceptance of Complementary and Integrative Practices in Health by a User Community of the Sistema Único de Saúde in the city of Tubarão/SC." Files catarineses of Medicine, 1806-4280/07/36-01/69. 


\section{Annex I The explanatory booklet}

\section{Homeopathy: How to Use}

- $\quad$ Take the medicine the way and the time determined by your homeopath doctor;

- $\quad$ Avoid direct contact with the medicine with your hands. To take your medicine use the bottle cap as a carrier;

- $\quad$ In case of liquid medicines in drops, avoid touching the bill in the mouth or tongue;

- $\quad$ In the case of medicinal products in hydro alcoholic solution, the drops may be administered in a small portion of filtered water;

- $\quad$ Do not drink alcohol along with the medicine;

- $\quad$ Never swallow the medicine after brushing your teeth or drinking coffee;

- $\quad$ All medicines should be kept out of the reach of children;

- In case of accidental ingestion of homeopathic medicine the doctor should be immediately notified;

- $\quad$ The homeopathic medicine is sensitive to strong odors, such as: sachets; perfumes. They should be kept away from these;

- $\quad$ Keep away from light, moisture and heat;

- $\quad$ The medicine should be stored in a dry and cool place;

- $\quad$ The bathroom is not suitable place to store medicines;

- $\quad$ The homeopathic medicine is sensitive to x-rays and other radiations. Conserve distant from television and radio sets.

\section{Important}

- $\quad$ Do not stop your treatment without telling your doctor;

- $\quad$ Always leave your medicines in the same place, so make it easy to remember to take them;

- $\quad$ Do not give your medicines to another person;

- $\quad$ In case of doubt about your prescription contact your doctor or pharmacist;

- $\quad$ If you feel anything different or strange, tell your doctor or pharmacist immediately. 